\title{
Characterization of matrix Fourier multiwavelet frames multipliers with integer dilation factor
}

\author{
Fengjuan Zhu, Yongdong Huang*, Shengnan Shi, Xiao Tan, Juan Zhao \\ School of Mathematics and Information Science, North Minzu University, Yinchuan, 750021, China.
}

Communicated by Y. H. Yao

\begin{abstract}
This paper investigates matrix Fourier multiwavelet frames multipliers with dilation factor a. First, the definition of matrix Fourier multiwavelet frame multiplier was proposed, which is $\mathrm{N}_{1} \times \mathrm{N}$ matrices with $\mathrm{L}^{\infty}$ function entries, and maps Parseval multiwavelet frames of length $\mathrm{N}$ to Parseval multiwavelet frames of length $\mathrm{N}_{1}$. Then, two sufficient conditions of matrix Fourier multiwavelet frame multiplier were given, and two necessary conditions of matrix Fourier multiwavelet frame multiplier were characterized by means of frame wavelet sets. Finally, several numerical examples were constructed. As Fourier wavelet frames multiplier, matrix Fourier multipliers can be used to derive new Parseval multiwavelet frames and can help us better understand the basic of frame theory. (c)2017 All rights reserved.
\end{abstract}

Keywords: Parseval frames multiwavelet, Fourier multipliers, matrix Fourier multiwavelet frames multipliers, frame wavelet set.

2010 MSC: 42C15, 42C40.

\section{Introduction}

As we all know, people have tried to use existing wavelets to construct new wavelets, and proposed the concept of wavelet multiplier and given a series of characterizations and results of wavelet multipliers $[6,8,13,16,18]$. Whether the wavelet multiplier (that can be seen as an operator) must be a unitary operator is still an open problem so far. However, a class of special wavelet multipliers-Fourier wavelet multiplier, gave a positive answer that Fourier wavelet multiplier must be a unit operator. Based on this good character of the Fourier wavelet multiplier, people naturally carried out systematic study and characterize it. Because of the rapid development of frame theory in nearly twenty years, people naturally want to use the given frame to get a new frame. Consequently, the concept of wavelet multipliers is generalized to wavelet frame multipliers, Parseval wavelet frame multipliers and Parseval multiwavelet frame multipliers, and then people gave the corresponding characterizations [1, 5, 10-12, 14, 15, 17]. Unfortunately, these references only consider the case of dilation factor 2 and force the matrix is a square matrix.

\footnotetext{
${ }^{*}$ Corresponding author

Email addresses: xiao_jinlin@163.com (Fengjuan Zhu), nxhyd74@126.com (Yongdong Huang), 977998177@qq.com (Shengnan Shi), 13649507784@163.com (Xiao Tan), 1334801264@qq.com (Juan Zhao)
}

doi:10.22436/jnsa.010.09.32

Received 2017-07-10 
In order to improve the theory of matrix Fourier frame wavelet multiplier and multi-channel theory, this paper will give the concept of matrix Fourier multiwavelet frames multipliers with dilation factor $a$, and obtain two sufficient conditions and two necessary conditions of the matrix Fourier multiwavelet frames multipliers with dilation factor a. Finally, we also give some numerical examples.

Let $\mathcal{H}$ be a separable Hilbert space. The sequence $\left\{\varphi_{k}\right\}_{k \in \Lambda}$ in $\mathcal{H}$ is called a frame if there exist constants $0<A \leqslant B<\infty$ such that

$$
A\|f\|^{2} \leqslant \sum_{k \in \Lambda}\left|<f, \varphi_{k}>\right|^{2} \leqslant B\|f\|^{2}, \quad \forall f \in \mathcal{H} .
$$

A, B are called the lower and upper frame bounds respectively.

If the upper and lower frame bounds are equal, then the frame is called tight frame. If it ceases to be a frame whenever any one of its elements is removed, the frame is called exact frame. In particular, frame $\left\{\varphi_{k}\right\}_{k \in t \wedge}$ is called Parseval frame when $A=B=1$, and there exists the following reconstruction formula

$$
f=\sum_{n \in \mathbf{Z}}\left\langle f, \varphi_{n}\right\rangle \varphi_{n} .
$$

Furthermore, if $\left\{\varphi_{k}\right\}_{k \in \Lambda}$ is also linear independence, then $\left\{\varphi_{k}\right\}_{k \in \Lambda}$ is called a Riesz basis for $\mathcal{H}$.

In this paper, let $\mathcal{H}=\mathrm{L}^{2}(\mathbb{R}), \psi \in \mathrm{L}^{2}(\mathbb{R})$, and

$$
D^{j} T^{k} \psi(t)=\psi_{j, k}(t)=a^{\frac{j}{2}} \psi\left(a^{j} t-k\right),
$$

where $a$ is called the dilation factor, i.e., $a$ is a positive integer that greater than or equal to 2 . For $f \in L^{1}(\mathbb{R}) \cap L^{2}(\mathbb{R})$, Fourier transform $\mathcal{F}$ is defined as follows:

$$
(\mathcal{F} f)(\xi)=\widehat{f}(\xi)=\frac{1}{\sqrt{2 \pi}} \int_{\mathbb{R}} f(x) e^{-i x \xi} d x .
$$

By the Plancheral theorem, the definition can be uniquely extended to $L^{2}(\mathbb{R})$. In this paper, $\mathcal{F}^{-1}$ represents inverse Fourier transform, $\chi_{E}$ represents characteristic function of measurable set $E, \emptyset$ denotes empty set, $|G|$ denotes measure of measurable set $G$.

If sequence $\left\{D^{j} T^{k} \psi, j, k \in \mathbb{Z}\right\}$ is a frame for $L^{2}(\mathbb{R})$ with the lower bound $A$ and upper frame bound $B$, then $\psi$ is called a frame wavelet and we have

$$
A\|f\|^{2} \leqslant \sum_{j \in \mathbb{Z}} \sum_{k \in \mathbb{Z}}\left|\left\langle f, \psi_{j, k}\right\rangle\right|^{2} \leqslant B\|f\|^{2} .
$$

Furthermore, if sequence $\left\{D^{j} T^{k} \psi, j, k \in \mathbb{Z}\right\}$ is a Parseval frame of $L^{2}(\mathbb{R})$, then $\psi$ is called Parseval frame wavelet. If sequence $\left\{D^{j} T^{k} \psi, j, k \in \mathbb{Z}\right\}$ is an orthonormal basis of $L^{2}(\mathbb{R})$, then $\psi$ is called orthonormal wavelet.

Definition 1.1 ([2]). Let $\psi^{\ell} \in \mathrm{L}^{2}(\mathbb{R}), \ell=1,2, \cdots, N$. Suppose that the $\operatorname{set}\left\{\left\{\psi_{j, k}^{\ell}\right\}_{j, k \in \mathbb{Z}}\right\}_{\ell=1}^{N}$ constitutes the Parseval frame for $\mathrm{L}^{2}(\mathbb{R})$, and

$$
\sum_{\ell=1}^{N} \sum_{j \in \mathbb{Z}} \sum_{k \in \mathbb{Z}}\left|\left\langle f, \psi_{j, k}^{\ell}\right\rangle\right|^{2}=\|f\|_{2}^{2}, \quad \forall f \in L^{2}(\mathbb{R}) .
$$

Then $\left(\psi^{1}, \psi^{2}, \cdots, \psi^{N}\right)$ is called Parseval frame multiwavelet or Parseval multiwavelet frame with dilation factor a of length $N$. In particular, if $\left\{\left\{\psi_{j, k}^{\ell}\right\}_{j, k \in \mathbb{Z}}\right\}_{\ell=1}^{N}$ can form an orthonormal basis for $L^{2}(\mathbb{R})$, then $\left(\psi^{1}, \psi^{2}, \cdots, \psi^{N}\right)$ is called orthonormal multiwavelet with dilation factor a.

We first give a characterization of Parseval Frame multiwavelet with dilation factor a. 
Proposition $1.2([2])$. Let $\psi^{\ell} \in \mathrm{L}^{2}(\mathbb{R}), \ell=1,2, \cdots, \mathrm{N}$. Then $\left(\psi^{1}, \psi^{2}, \cdots, \psi^{\mathrm{N}}\right)$ is a Parseval frame multiwavelet with dilation factor a of length $\mathrm{N}$ if and only if

$$
\sum_{\ell=1}^{N} \sum_{j \in \mathbb{Z}}\left|\widehat{\psi^{\ell}}\left(a^{j} \xi\right)\right|^{2}=\frac{1}{2 \pi} \text {, a.e. } \xi \in \mathbb{R},
$$

and

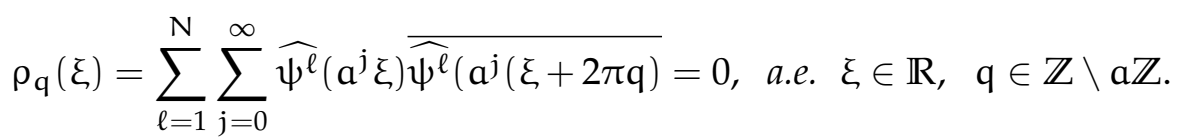

Suppose that $f$ is a measurable function, for any Parseval frame wavelet $\psi$, if the inverse fourier transform of $(f \hat{\psi})$ is always Parseval frame wavelet, then $f$ is called Fourier wavelet frame multiplier. For Fourier wavelet frame multiplier, we have the following conclusion:

Proposition 1.3 ([16, 18]). A measurable function $\mathrm{f}$ is a Fourier wavelet frames multiplier with dilation factor 2 if and only if $\|\mathrm{f}\|=1$ and the period of $\mathrm{f}(2 \mathrm{~s}) / \mathrm{f}(\mathrm{s})$ is $2 \pi$.

The definition of the matrix Fourier wavelet frames multiplier with dilation factor 2 in [14] is as follows.

Proposition 1.4 ([14]). Assume that $m_{i j}(\xi), i, j=1,2, \cdots, N$ are measurable function, $M(\xi)=\left(m_{i j}(\xi)\right)_{N \times N}$ is a matrix-value function. For any Parseval frame multiwavelets $\left(\psi^{1}, \psi^{2}, \ldots, \psi^{\mathrm{N}}\right)$ with dilation factor 2 , if the inverse Fourier transform of $\mathrm{M}(\xi)\left(\widehat{\psi^{1}}(\xi), \widehat{\psi^{2}}(\xi), \cdots, \widehat{\psi^{N}}(\xi)\right)^{\top}=\left(\widehat{\eta^{1}}(\xi), \widehat{\eta^{2}}(\xi), \cdots, \widehat{\eta^{N}}(\xi)\right)^{\top}$ is also a Parseval frame multiwavelet with dilation factor 2 , then $\mathrm{M}(\xi)=\left(\mathrm{m}_{i, j}(\xi)\right)_{\mathrm{N} \times \mathrm{N}}$ is called matrix Fourier multiwavelet frames multiplier with dilation factor 2.

In fact, we find it is not necessary to limit that $M(\xi)$ is a square matrix. Here we give two examples to illustrate this fact.

Example 1.5. When $N=1$, let $M_{1}(\xi)=(\cos (\xi), \sin (\xi))^{\top}, M_{2}(\xi)=(\cos (\xi), \sin (\xi) \cos (\theta), \sin (\xi) \sin (\theta))^{\top}$, $\theta \in \mathbb{R}$. Assume that $\psi$ is any Parseval frame multiwavelets with dilation factor a of length 1 , let $\left(\widehat{\psi^{1}}(\xi), \widehat{\psi^{2}}(\xi)\right)^{\top}=M_{1}(\xi) \widehat{\psi}(\xi)$, i.e., $\widehat{\psi^{1}}(\xi)=\cos (\xi) \widehat{\psi}(\xi), \widehat{\psi^{2}}(\xi)=\sin (\xi) \widehat{\psi}(\xi)$. Then, we have

$$
\sum_{\ell=1}^{2} \sum_{j \in \mathbb{Z}}\left|\widehat{\psi^{\ell}}\left(a^{j} \xi\right)\right|^{2}=\sum_{j \in \mathbb{Z}}\left|\widehat{\psi}\left(a^{j} \xi\right)\right|^{2}=\frac{1}{2 \pi}, \text { a.e. } \xi \in \mathbb{R},
$$

and

$$
\rho_{\mathrm{q}}(\xi)=\sum_{\ell=1}^{2} \sum_{j=0}^{\infty} \widehat{\psi^{\ell}}\left(a^{j} \xi\right) \overline{\psi^{\ell}}\left(a^{j}(\xi+2 \pi q)\right)=\sum_{j=0}^{\infty} \widehat{\psi}\left(a^{j} \xi\right) \widehat{\psi^{\ell}\left(a^{j}(\xi+2 \pi q)\right)}=0, \text { a.e. } \xi \in \mathbb{R}, \quad q \in \mathbb{Z} \backslash a \mathbb{Z} .
$$

According to Proposition 1.2, $\left(\psi^{1}, \psi^{2}\right)$ is a Parseval frame multiwavelet with dilation factor a of length 2. In similar way, the inverse fourier transform of $M_{2}(\xi) \widehat{\psi}(\xi)$ is a Parseval frame multiwavelet with dilation factor $a$ of length 3 , where $a \geqslant 2$.

Example 1.6. When $N=2$, let $M_{3}(\xi)=\left(\begin{array}{ll}a_{11} & a_{12} \\ a_{21} & a_{22}\end{array}\right)$ is a unitary matrix. According to [14], we know $M_{3}(\xi)$ is a matrix Fourier multiwavelet frame multiplier with dilation factor 2. Furthermore, let $M_{4}(\xi)=$ $\left(\begin{array}{cc}\cos (\xi) & \sin (\xi) \\ \sin (\xi) \sin (\theta) & -\cos (\xi) \sin (\theta) \\ \sin (\xi) \cos (\theta) & -\cos (\xi) \cos (\theta)\end{array}\right)$. Suppose that $\left(\psi^{1}, \psi^{2}\right)$ is a Parseval frame multiwavelet with dilation factor a of length 2 . Let $M_{4}(\xi)\left(\widehat{\psi^{1}}(\xi), \widehat{\psi^{2}}(\xi)\right)^{\top}=\left(\widehat{\eta^{1}}(\xi), \widehat{\eta^{2}}(\xi), \widehat{\eta^{3}}(\xi)\right)^{\top}$, we have

$$
\sum_{\ell=1}^{3} \sum_{j \in \mathbb{Z}}\left|\widehat{\eta}^{\ell}\left(a^{j} \xi\right)\right|^{2}=\sum_{\ell=1}^{2} \sum_{j \in \mathbb{Z}}\left|\widehat{\psi^{\ell}}\left(a^{j} \xi\right)\right|^{2}=\frac{1}{2 \pi^{\prime}} \text {, a.e. } \xi \in \mathbb{R},
$$


and

$$
\sum_{\ell=1}^{3} \sum_{j=0}^{\infty} \widehat{\eta^{\ell}}\left(a^{j} \xi\right) \overline{\bar{\psi}^{\ell}\left(a^{j}(\xi+2 \pi q)\right)}=\sum_{\ell=1}^{2} \sum_{j=0}^{\infty} \widehat{\psi^{\ell}}\left(a^{j} \xi\right) \overline{\widehat{\psi^{\ell}}\left(a^{j}(\xi+2 \pi q)\right)}=0, \text { a.e. } \xi \in \mathbb{R}, \quad q \in \mathbb{Z} \backslash a \mathbb{Z} .
$$

By Proposition 1.2, $\left(\eta^{1}, \eta^{2}, \eta^{3}\right)$ is a Parseval frame multiwavelet with dilation factor a of length 3 , where $a \geqslant 2$.

From Examples 1.5 and 1.6, $M_{1}(\xi), M_{2}(\xi)$ and $M_{4}(\xi)$ can act as matrix Fourier multiwavelet frame multipliers. Based on this fact, we give the following definition.

Definition 1.7. Assume that $m_{i j}(\xi), i=1,2, \cdots, N_{1}, j=1,2, \cdots, N$ are measurable function, $M(\xi)=$ $\left(m_{i j}(\xi)\right)_{N_{1} \times N}$ is a matrix-value function and $N_{1} \geqslant N$. For any Parseval frame multiwavelet $\left(\psi^{1}, \psi^{2}, \ldots, \psi^{N}\right)$ with dilation factor $a$, if the inverse Fourier transform of

$$
M(\xi)\left(\widehat{\psi^{1}}(\xi), \widehat{\psi^{2}}(\xi), \cdots, \widehat{\psi^{N}}(\xi)\right)^{\top}=\left(\widehat{\eta^{1}}(\xi), \widehat{\eta}^{2}(\xi), \cdots, \widehat{\eta^{N_{1}}}(\xi)\right)^{\top},
$$

is also a Parseval frame multiwavelet with dilation factor $a$, then $M(\xi)=\left(m_{i, j}(\xi)\right)_{N_{1} \times N}$ is called matrix Fourier multiwavelet frames multiplier with dilation factor $a$.

\section{Main conclusions and proofs}

From Example 1.5 and Example 1.6, we have

$$
M_{1}^{*}(\xi) M_{1}(\xi)=M_{2}^{*}(\xi) M_{2}(\xi)=1, \quad M_{3}^{*}(\xi) M_{3}(\xi)=M_{4}^{*}(\xi) M_{4}(\xi)=I_{2} .
$$

We first give two sufficient conditions of matrix Fourier multiwavelet frames multipliers with dilation factor a.

Theorem 2.1. Assume that $m_{i, j}(\xi) \in L^{\infty}(\mathbb{R}), i=1,2, \cdots, N_{1}, j=1,2, \cdots, N, M(\xi)=\left(m_{i, j}(\xi)\right)_{N_{1} \times N}$ is a matrix-value function and $\mathrm{N}_{1}>\mathrm{N}$. If $\mathrm{M}(\xi)$ satisfies the following conditions:

(1) $M^{*}(\xi) M(\xi)=I_{N}$ for $\xi \in \mathbb{R}$;

(2) $M(a \xi) M^{*}(\xi)$ is $2 \pi$-periodic function;

(3) $M^{*}(\xi) M(\xi+2 \pi q)=\lambda_{q}(\xi) I_{N}$, where $\lambda_{q}(\xi)=1, q \in \mathbb{Z} \backslash a \mathbb{Z}$,

then $\mathrm{M}(\xi)$ is a matrix Fourier multiwavelet frames multiplier with dilation factor a.

Proof. For any Parseval frame multiwavelet $\left(\psi^{1}, \psi^{2}, \cdots, \psi^{N}\right)$, let

$$
\left(\begin{array}{c}
\widehat{\eta^{1}}(\xi) \\
\widehat{\eta^{2}}(\xi) \\
\vdots \\
\widehat{\eta^{N_{1}}}(\xi)
\end{array}\right)=M(\xi)\left(\begin{array}{c}
\widehat{\psi^{1}}(\xi) \\
\widehat{\psi^{2}}(\xi) \\
\vdots \\
\widehat{\psi^{N}}(\xi)
\end{array}\right)
$$

The proof is divided into four steps:

The first step: since $M^{*}(\xi) M(\xi)=I_{N}$, we have that

$$
\sum_{\ell=1}^{N_{1}} \sum_{j \in \mathbb{Z}}\left|\widehat{\eta}^{\ell}\left(a^{j} \xi\right)\right|^{2}=\sum_{j \in \mathbb{Z}}\left\langle M\left(a^{j} \xi\right)\left(\begin{array}{c}
\widehat{\psi^{1}}\left(a^{j} \xi\right) \\
\vdots \\
\widehat{\psi^{N}}\left(a^{j} \xi\right)
\end{array}\right), M\left(a^{j} \xi\right)\left(\begin{array}{c}
\widehat{\psi^{1}}\left(a^{j} \xi\right) \\
\vdots \\
\widehat{\psi^{N}}\left(a^{j} \xi\right)
\end{array}\right)\right\rangle
$$




$$
\begin{aligned}
& =\sum_{j \in \mathbb{Z}}\left\langle\left(\begin{array}{c}
\widehat{\psi}^{1}\left(a^{j} \xi\right) \\
\vdots \\
\widehat{\psi^{N}}\left(a^{j} \xi\right)
\end{array}\right), M^{*}\left(a^{j} \xi\right) M\left(a^{j} \xi\right)\left(\begin{array}{c}
\widehat{\psi^{1}}\left(a^{j} \xi\right) \\
\vdots \\
\widehat{\psi^{N}}\left(a^{j} \xi\right)
\end{array}\right)\right\rangle \\
& =\sum_{j \in \mathbb{Z}} \sum_{\ell=1}^{N}\left|\widehat{\psi^{\ell}}\left(a^{j} \xi\right)\right|^{2}=\sum_{\ell=1}^{N} \sum_{j \in \mathbb{Z}}\left|\widehat{\psi^{\ell}}\left(a^{j} \xi\right)\right|^{2}=\frac{1}{2 \pi} .
\end{aligned}
$$

Therefore, the formula (1.1) of Proposition 1.2 holds.

The second step: when $j \geqslant 1$, let $t=a^{j-1} \xi$. For $q \in \mathbb{Z} \backslash a \mathbb{Z}$, from the condition (2) of Theorem 2.1, we have that

$$
\begin{aligned}
M\left(a^{j} \xi\right) M^{*}\left(a^{j-1} \xi\right) & =M\left(a\left(a^{j-1} \xi\right)\right) M^{*}\left(a^{j-1} \xi\right)=M(a t) M^{*}(t) \\
& =M\left(a\left(t+a^{j-1} 2 \pi q\right)\right) M^{*}\left(t+a^{j-1} 2 \pi q\right) \\
& =M\left(a\left(a^{j-1} \xi+a^{j-1} 2 \pi q\right)\right) M^{*}\left(a^{j-1} \xi+a^{j-1} 2 \pi q\right) \\
& =M\left(a^{j}(\xi+2 \pi q)\right) M^{*}\left(a^{j-1}(\xi+2 \pi q)\right) .
\end{aligned}
$$

The third step: due to $M^{*}(\xi) M(\xi)=I_{N}$, by matrix extension principle, there exists an $N_{1} \times N_{1}$ unitary matrix $U(\xi)$ such that

$$
\mathrm{M}(\xi)=\mathrm{U}(\xi)\left(\begin{array}{c}
\mathrm{I}_{\mathrm{N}} \\
\mathrm{O}
\end{array}\right), \quad \mathrm{M}^{*}(\xi)=\left(\mathrm{I}_{\mathrm{N}}, \mathrm{O}\right) \mathrm{U}^{*}(\xi),
$$

where $\mathrm{O}$ denotes zero matrix with appropriate size. Using (2.1), we have

$$
U\left(a^{j} \xi\right)\left(\begin{array}{c}
I_{N} \\
O
\end{array}\right)\left(I_{N}, O\right) U^{*}\left(a^{j-1} \xi\right)=U\left(a^{j}(\xi+2 \pi q)\right)\left(\begin{array}{c}
I_{N} \\
O
\end{array}\right)\left(I_{N}, O\right) U^{*}\left(a^{j-1}(\xi+2 \pi q)\right) .
$$

Since both $U\left(a^{j} \xi\right)$ and $U^{*}\left(a^{j-1}(\xi+2 \pi q)\right)$ are unitary matrix, $U^{*}\left(a^{j} \xi\right)$ premultiplying both sides of (2.2) and $U\left(a^{j-1}(\xi+2 \pi q)\right)$ postmultiplying the both sides of $(2.2)$, respectively. We obtain that

$$
\left(\begin{array}{c}
I_{N} \\
O
\end{array}\right)\left(I_{N}, O\right) U^{*}\left(a^{j-1} \xi\right) U\left(a^{j-1}(\xi+2 \pi q)\right)=U^{*}\left(a^{j} \xi\right) U\left(a^{j}(\xi+2 \pi q)\right)\left(\begin{array}{c}
I_{N} \\
O
\end{array}\right)\left(I_{N}, O\right) .
$$

Now, $\left(\mathrm{I}_{\mathrm{N}}, \mathrm{O}\right)$ premultiplying both sides of $(2.3)$ and $\left(\begin{array}{c}\mathrm{I}_{\mathrm{N}} \\ \mathrm{O}\end{array}\right)$ postmultiplying the both sides of (2.3), respectively. We obtain that

$$
\left(I_{N}, O\right) U^{*}\left(a^{j-1} \xi\right) U\left(a^{j-1}(\xi+2 \pi q)\right)\left(\begin{array}{c}
I_{N} \\
O
\end{array}\right)=\left(I_{N}, O\right) U^{*}\left(a^{j} \xi\right) U\left(a^{j}(\xi+2 \pi q)\right)\left(\begin{array}{c}
I_{N} \\
O
\end{array}\right)
$$

Thus

$$
M^{*}\left(a^{j} \xi\right) M\left(a^{j}(\xi+2 \pi q)\right)=M^{*}\left(a^{j-1} \xi\right) M\left(a^{j-1}(\xi+2 \pi q)\right) .
$$

Repeat use of (2.4), we have

$$
\begin{aligned}
M^{*}\left(a^{j} \xi\right) M\left(a^{j}(\xi+2 \pi q)\right) & =M^{*}\left(a^{j-1} \xi\right) M\left(a^{j-1}(\xi+2 \pi q)\right) \\
& =M^{*}\left(a^{j-2} \xi\right) M\left(a^{j-2}(\xi+2 \pi q)\right) \\
& =\cdots=M^{*}(\xi) M(\xi+2 \pi q)=\lambda_{q}(\xi) .
\end{aligned}
$$

The fourth step: let

$$
\rho_{q}(\xi)=\sum_{\ell=1}^{N_{1}} \sum_{j=0}^{\infty} \widehat{\eta^{\ell}}\left(a^{j} \xi\right) \overline{\eta^{\ell}\left(a^{j}(\xi+2 \pi q)\right)}, q \in \mathbb{Z} \backslash a \mathbb{Z}
$$


From (2.4), for $q \in \mathbb{Z} \backslash a \mathbb{Z}$, it follows that

$$
\begin{aligned}
& \rho_{q}(\xi)=\sum_{j=0}^{\infty}\left\langle M\left(a^{j} \xi\right)\left(\begin{array}{c}
\widehat{\psi^{1}}\left(a^{j} \xi\right) \\
\vdots \\
\widehat{\psi^{N}}\left(a^{j} \xi\right)
\end{array}\right), M\left(a^{j}(\xi+2 \pi q)\right)\left(\begin{array}{c}
\widehat{\psi^{1}}\left(a^{j}(\xi+2 \pi q)\right) \\
\vdots \\
\widehat{\psi^{N}}\left(a^{j}(\xi+2 \pi q)\right)
\end{array}\right)\right\rangle \\
& =\sum_{j=0}^{\infty}\left\langle\left(\begin{array}{c}
\widehat{\psi^{1}}\left(a^{j} \xi\right) \\
\vdots \\
\widehat{\psi^{N}}\left(a^{j} \xi\right)
\end{array}\right), M^{*}\left(a^{j} \xi\right) M\left(a^{j}(\xi+2 \pi q)\right)\left(\begin{array}{c}
\widehat{\psi^{1}}\left(a^{j}(\xi+2 \pi q)\right) \\
\vdots \\
\widehat{\psi^{N}}\left(a^{j}(\xi+2 \pi q)\right)
\end{array}\right)\right\rangle \\
& =\overline{\lambda_{q}(\xi)} \sum_{j=0}^{\infty}\left\langle\left(\begin{array}{c}
\widehat{\psi^{1}}\left(a^{j} \xi\right) \\
\vdots \\
\widehat{\psi^{N}}\left(a^{j} \xi\right)
\end{array}\right),\left(\begin{array}{c}
\widehat{\psi^{1}}\left(a^{j}(\xi+2 \pi q)\right) \\
\widehat{\psi^{N}}\left(a^{j}(\xi+2 \pi q)\right)
\end{array}\right)\right\rangle \\
& =\overline{\lambda_{q}(\xi)} \sum_{j=0}^{\infty} \sum_{\ell=1}^{N} \widehat{\psi^{\ell}}\left(a^{j} \xi\right) \overline{\psi^{\ell}\left(a^{j}(\xi+2 \pi q)\right)}=\overline{\lambda_{q}(\xi)} \sum_{\ell=1}^{N} \sum_{j=0}^{\infty} \widehat{\psi^{\ell}}\left(a^{j} \xi\right) \overline{\psi^{\ell}}\left(a^{j}(\xi+2 \pi q)\right)=0 .
\end{aligned}
$$

Hence, equation (1.2) of Proposition 1.2 holds. Therefore, $M(\xi)$ is a matrix Fourier frame multiwavelet multiplier with dilation factor a.

Remark 2.2. The third step of the proof of Theorem 2.1 needs using the matrix extension principle, but if you adopt the method of the literature [14], we cannot get the result of Theorem 2.1. This is due to the multiplier in the literature [14] is a unitary matrix, but the multiplier $M(\xi)$ of Theorem 2.1 is a more general matrix.

Theorem 2.3. Suppose that $m_{i, j}(\xi) \in L^{\infty}(\mathbb{R}), i=1,2, \cdots, N_{1}, j=1,2, \cdots, N, M(\xi)=\left(m_{i, j}(\xi)\right)_{N_{1} \times N}$ is a matrix-valued function and $\mathrm{N}_{1} \geqslant \mathrm{~N}$. If $\mathrm{M}(\xi)$ satisfies the following conditions

(1) $M^{*}(\xi) M(\xi)=I_{N}$ for $\xi \in \mathbb{R}$;

(2) $\mathrm{M}(\xi)$ is $2 \pi$-periodical,

then $\mathrm{M}(\xi)$ is a matrix Fourier frame multiwavelet multiplier with dilation factor a.

Proof. The proof of Theorem 2.3 is similar to the proof of Theorem 2.1.

Remark 2.4. When $\mathrm{N}=1, \mathrm{a}=3$, let $\mathrm{M}_{1}(\xi)=\left(\frac{1}{\sqrt{2}} e^{-\frac{\mathrm{i} \xi}{2}}, \frac{1}{\sqrt{2}} e^{\frac{i \xi}{2}}\right)^{\top}$. Let $\psi$ be any Parseval frame multiwavelet with dilation factor 3 of length 1 , and $\left(\widehat{\psi^{1}}(\xi), \widehat{\psi^{2}}(\xi)\right)^{\top}=M_{1}(\xi) \widehat{\psi}(\xi)$, that is $\widehat{\psi^{1}}(\xi)=\frac{1}{\sqrt{2}} e^{-\frac{i \xi}{2}} \widehat{\psi}(\xi), \widehat{\psi^{2}}(\xi)=$ $\frac{1}{\sqrt{2}} e^{\frac{i \xi}{2}} \widehat{\psi}(\xi)$. After simple calculation, we have

$$
\sum_{\ell=1}^{2} \sum_{j \in \mathbb{Z}}\left|\widehat{\psi^{\ell}}\left(3^{j} \xi\right)\right|^{2}=\sum_{j \in \mathbb{Z}}\left|\widehat{\psi}\left(3^{j} \xi\right)\right|^{2}=\frac{1}{2 \pi^{\prime}} \text { a.e. } \xi \in \mathbb{R},
$$

and

$$
\left.\rho_{\mathrm{q}}(\xi)=\sum_{\ell=1}^{2} \sum_{j=0}^{\infty} \widehat{\psi^{\ell}}\left(3^{j} \xi\right) \overline{\widehat{\psi^{\ell}}\left(3^{j}(\xi+2 \pi q)\right.}\right)=(-1)^{q} \sum_{j=0}^{\infty} \widehat{\psi}\left(3^{j} \xi\right) \overline{\widehat{\psi^{\ell}}\left(3^{j}(\xi+2 \pi q)\right)}=0, \quad q \in \mathbb{Z} \backslash 3 \mathbb{Z} .
$$

That is, $M(\xi)$ is a matrix Fourier frame multiwavelet multiplier with dilation factor 3. In fact, $M(\xi)$ satisfies three conditions of Theorem 2.1, but $M(\xi)$ does not satisfy the conditions of Theorem 2.3. In fact, $M(\xi)$ is not $2 \pi$-periodical. 
Remark 2.5. When the condition $M^{*}(\xi) M(\xi)=I_{N}$ holds, the condition (2) of Theorem 2.3 can derive the conditions (2) and (3) of Theorem 2.1. Conversely, the conditions (2) and (3) of Theorem $2.1 \mathrm{might}$ not yield the condition (2) of Theorem 2.3. This states the condition " $M(\xi)$ is $2 \pi$-periodical" is not the necessary condition of " $\mathrm{M}(\xi)$ is matrix Fourier frame multiwavelet multiplier with dilation factor $a$ ".

In order to discuss the necessary conditions of the matrix Fourier frame multiwavelet multiplier, we firstly review the concept of wavelet set and frame wavelet sets. Let $E$ be a measurable subset of $\mathbb{R}$. If the inverse Fourier transform of $\hat{\psi}(\xi)=\frac{1}{\sqrt{2 \pi}} \chi_{E}$ is an orthonormal wavelet, then $E$ is called a wavelet set. Similarly, if the inverse Fourier transform of $\hat{\psi}(\xi)=\frac{1}{\sqrt{2 \pi}} \chi_{E}$ is a Parseval Frame wavelet, then $E$ is called a Parseval frame wavelet set. $E$ is a wavelet set with dilation factor $a$ if and only if $|E|=2 \pi,\{E+2 \ell \pi: \ell \in \mathbb{Z}\}$ and $\left\{a^{n} E: n \in \mathbb{Z}\right\}$ form a disjoint partition of $\mathbb{R}$. The conclusions of wavelet sets and frame wavelet sets are given in $[3,4,7,9]$.

Lemma 2.6. Let a be any positive integer dilation factor, and $\mathrm{G}=\mathrm{A} \cup \mathrm{B} \cup \mathrm{C} \cup \mathrm{D}$, where

$$
A=\left[-\frac{2 a \pi}{a+1},-\frac{2 \pi}{a+1}\right), \quad B=\left[\frac{2 \pi}{a^{2}-1}, \frac{2 \pi}{a+1}\right), \quad C=\left[\frac{2 a \pi}{a+1}, 2 \pi\right), \quad D=\left[2 \pi, \frac{2 a^{2} \pi}{a^{2}-1}\right),
$$

then $\mathrm{G}$ is a wavelet set with dilation factor $\mathrm{a}$.

Proof. Firstly, we know

$$
\begin{aligned}
(A+2 \pi) \cap B & =(A+2 \pi) \cap C=B \cap C=(A+2 \pi) \cap(D-2 \pi) \\
& =B \cap(D-2 \pi)=C \cap(D-2 \pi)=\emptyset,
\end{aligned}
$$

and

$$
(A+2 \pi) \cup B \cup C \cup(D-2 \pi)=[0,2 \pi) .
$$

In addition, $|G|=2 \pi$. So $\bigcup_{k \in \mathbb{Z}}(G+2 k \pi)=\mathbb{R}$.

Secondly, $\left\{A, B, \frac{1}{a} C, \frac{1}{a} D\right\}$ is a partition of set $\left[-\frac{2 a \pi}{a+1},-\frac{2 \pi}{a+1}\right) \bigcup\left[\frac{2 \pi}{a^{2}-1}, \frac{2 a \pi}{a^{2}-1}\right)$. Therefore, $\left\{a^{n} G: n \in \mathbb{Z}\right\}$ is a partition of $\mathbb{R}$. Finally, let $\psi=\frac{1}{\sqrt{2 \pi}} \mathcal{F}^{-1}\left(\chi_{G}\right)$, then $\left\{a^{\frac{n}{2}} \psi\left(a^{n} t-\ell\right): n, \ell \in \mathbb{Z}\right\}$ constitutes the orthonormal bases of $L^{2}(\mathbb{R})$. In summary, $G$ is a wavelet set with dilation factor a and $\psi$ is an orthonormal wavelet with dilation factor $a$.

Based on Lemma 2.6, we can get the following conclusion.

Lemma 2.7. Let $\mathrm{a}$ be any positive integer dilation factor, then there exist $\mathrm{N}$ measurable sets $\mathrm{E}_{1}, \mathrm{E}_{2}, \cdots, \mathrm{E}_{\mathrm{N}}$ with measure of nonzero, which satisfy the following conditions:

(1) for any $\ell, k \in \mathbb{Z}$, we have $\left(E_{i}+2 \ell \pi\right) \cap\left(E_{j}+2 k \pi\right)=\emptyset, i \neq j, i, j=1,2, \cdots, N$;

(2) let $\mathrm{G}=\mathrm{E}_{1} \cup \mathrm{E}_{2} \bigcup \cdots \cup \mathrm{E}_{\mathrm{N}}$, then $|\mathrm{G}|=2 \pi$ and $\bigcup_{\mathrm{k} \in \mathbb{Z}}(\mathrm{G}+2 \mathrm{k} \pi)=\mathbb{R}$;

(3) $\left\{\mathrm{a}^{\mathrm{n}} \mathrm{G}: \mathrm{n} \in \mathbb{Z}\right\}$ constitutes a partition of $\mathbb{R}$. Furthermore, if let $\psi^{\mathrm{i}}:=\frac{1}{\sqrt{2 \pi}} \mathcal{F}^{-1}\left(\chi_{\mathrm{E}_{i}}\right)$, then $\left(\psi^{1}, \psi^{2}, \cdots, \psi^{\mathrm{N}}\right)$ is a Parseval frame multiwavelet with dilation factor a.

Proof. It is directly derived from Lemma 2.6 and Proposition 1.2.

Remark 2.8. Lemma 2.6 and Lemma 2.7 are very useful to characterize the necessary conditions of matrix fourier multiwavelet frames multipliers with dilation factor a. Because it ensures the existence of Parseval frame multiwavelets with dilation factor $a$.

According to [7, Corollary 3], we get the following lemma. 
Lemma 2.9 ([7]). Each measurable subset of $\mathbb{R}$ is a countable union of intersection of pairs of wavelet sets with dilation factor $a$. The family of wavelet sets with dilation factor a generates the Borel structure of $\mathbb{R}$.

Lemma 2.10. Assume $\xi \in \mathbb{R}, q$ is a nonzero integer. If $\xi \notin 2 \pi q \mathbb{Z}$ and $\xi \neq \frac{2 \pi q}{a^{j}-1}, j \neq 0, j \in \mathbb{Z}$, then there exists $\varepsilon>0$ such that $\xi+2 \pi q \notin \Omega=\bigcup_{n \in \mathbb{Z}} a^{n}[\xi-\varepsilon, \xi+\varepsilon]$.

Proof. The proof of Lemma 2.10 is similar to [14, Lemma 2.1].

Lemma 2.11. Assume that $m_{i, j}(\xi) \in L^{\infty}(\mathbb{R}), i=1,2, \cdots, N_{1}, j=1,2, \cdots, N, M(\xi)=\left(m_{i, j}(\xi)\right)_{N_{1} \times N}$ is a matrix-value function and $\mathrm{N}_{1} \geqslant \mathrm{~N}$. If $\mathrm{M}(\xi)$ is a matrix Fourier multiwavelet frames multiplier with dilation factor a, then $\mathrm{M}^{*}(\xi) \mathrm{M}(\xi)=\mathrm{I}_{\mathrm{N}}$.

Proof. Let $\left(\psi^{1}, \psi^{2}, \ldots, \psi^{N}\right)$ be a Parseval frame multiwavelet with dilation factor a, then $\left(\eta^{1}, \eta^{2}, \ldots, \eta^{N_{1}}\right)$ is also a Parseval frame multiwavelet with dilation factor $a$, where $M(\xi)\left(\begin{array}{c}\widehat{\psi^{1}}(\xi) \\ \vdots \\ \widehat{\psi^{N}}(\xi)\end{array}\right)=\left(\begin{array}{c}\widehat{\eta^{1}}(\xi) \\ \vdots \\ \widehat{\eta^{N_{1}}}(\xi)\end{array}\right)$. Using Proposition 1.2, for any $\xi \in \mathbb{R}$, we have

$$
\sum_{\ell=1}^{N_{1}} \sum_{j \in \mathbb{Z}}\left|\widehat{\eta}^{\ell}\left(a^{j} \xi\right)\right|^{2}=\sum_{j \in \mathbb{Z}}\left\langle\left(\begin{array}{c}
\widehat{\psi^{1}}\left(a^{j} \xi\right) \\
\vdots \\
\widehat{\psi^{N}}\left(a^{j} \xi\right)
\end{array}\right), M^{*}\left(a^{j} \xi\right) M\left(a^{j} \xi\right)\left(\begin{array}{c}
\widehat{\psi^{1}}\left(a^{j} \xi\right) \\
\vdots \\
\widehat{\psi^{N}}\left(a^{j} \xi\right)
\end{array}\right)\right\rangle=\frac{1}{2 \pi^{\prime}}
$$

and

$$
\sum_{\ell=1}^{N} \sum_{j \in \mathbb{Z}}\left|\widehat{\psi^{\ell}}\left(a^{j} \xi\right)\right|^{2}=\sum_{j \in \mathbb{Z}}\left\langle\left(\begin{array}{c}
\widehat{\psi^{1}}\left(a^{j} \xi\right) \\
\vdots \\
\widehat{\psi^{N}}\left(a^{j} \xi\right)
\end{array}\right),\left(\begin{array}{c}
\widehat{\psi^{1}}\left(a^{j} \xi\right) \\
\vdots \\
\widehat{\psi^{N}}\left(a^{j} \xi\right)
\end{array}\right)\right\rangle=\frac{1}{2 \pi} .
$$

By (2.5) and (2.6), for any $\xi \in \mathbb{R}$,

$$
\sum_{j \in \mathbb{Z}}\left\langle\left(\begin{array}{c}
\widehat{\psi^{1}}\left(a^{j} \xi\right) \\
\vdots \\
\widehat{\psi^{N}}\left(a^{j} \xi\right)
\end{array}\right),\left(I-M^{*}\left(a^{j} \xi\right) M\left(a^{j} \xi\right)\right)\left(\begin{array}{c}
\widehat{\psi^{1}}\left(a^{j} \xi\right) \\
\vdots \\
\widehat{\psi^{N}}\left(a^{j} \xi\right)
\end{array}\right)\right\rangle=0 .
$$

Next, we choose some special Parseval frame multiwavelets $\left(\psi^{1}, \psi^{2}, \cdots, \psi^{N}\right)$ with dilation factor a to ensure that $M^{*}(\xi) M(\xi)=I_{N}$.

For any nonzero $\xi \in \mathbb{R}$, there exists a wavelet set $G=E_{1} \cup E_{2} \cup \cdots \cup E_{N}$ with dilation factor a such that $\xi \in \mathrm{G}$, where $E_{i}$ satisfies the conditions of Lemma 2.7. Let $\psi^{i}$ be defined by the way of Lemma 2.7, then $\left\{\psi^{1}, \psi^{2}, \ldots, \psi^{N}\right\}$ is a Parseval frame multiwavelet with dilation factor a.

Because of $\xi \in G$, so there exists $i_{0}$, such that $\xi \in E_{i_{0}}$. Without loss of generality, let $i_{0}=1$, then $\xi \in E_{1}$ and $\xi \notin a^{j} E_{1}, j \neq 0$. At the same time, $\xi \notin a^{j} E_{i}, j \in \mathbb{Z}, i=2,3, \cdots, N$, so (2.7) becomes

$$
\left\langle\overrightarrow{b_{1}},\left(I-M^{*}(\xi) M(\xi)\right) \overrightarrow{b_{1}}\right\rangle=0,
$$

where $\overrightarrow{b_{1}}=(1,0, \cdots, 0)^{\top}$. Similarly, $\left\{\psi^{2}, \psi^{1}, \psi^{3}, \ldots, \psi^{N}\right\}$ is also a Parseval frame multiwavelet with dilation factor $a$, then

$$
\left\langle\overrightarrow{b_{2}},\left(I-M^{*}(\xi) M(\xi)\right) \overrightarrow{b_{2}}\right\rangle=0, \quad \overrightarrow{b_{2}}=(0,1,0, \cdots, 0)^{\top},
$$

and so on, we have

$$
\left\langle\overrightarrow{b_{N}},\left(I-M^{*}(\xi) M(\xi)\right) \overrightarrow{b_{N}}\right\rangle=0, \quad \overrightarrow{b_{N}}=(0, \cdots, 0,1)^{\top} .
$$

Namely, for any $N$-dimensional vector $\vec{b}$, we have

$$
\left\langle\vec{b},\left(I-M^{*}(\xi) M(\xi)\right) \vec{b}\right\rangle=0 .
$$

Therefore, $M^{*}(\xi) M(\xi)=I_{N}$. 
Theorem 2.12. Assume that $m_{i, j}(\xi) \in L^{\infty}(\mathbb{R}), i=1,2, \cdots, N_{1}, j=1,2, \cdots, N, M(\xi)=\left(m_{i, j}(\xi)\right)_{N_{1} \times N}$ is a matrix-value function and $\mathrm{N}_{1} \geqslant \mathrm{~N}$. If $\mathrm{M}(\xi)$ is a matrix Fourier multiwavelet frames multiplier with dilation factor $a$, then when $j \geqslant 0, q \in \mathbb{Z} \backslash a \mathbb{Z}, \xi \in \mathbb{R}$, we have $M^{*}\left(a^{j} \xi\right) M\left(a^{j}(\xi+2 q \pi)\right)$ is a diagonal matrix.

Proof. Let

$$
M^{*}\left(a^{j} \xi\right) M\left(a^{j}(\xi+2 q \pi)\right)=\left(\begin{array}{cccc}
f_{11}^{j}(\xi) & f_{12}^{j}(\xi) & \cdots & f_{1 N}^{j}(\xi) \\
f_{21}^{j}(\xi) & f_{22}^{j}(\xi) & \cdots & f_{2 N}^{j}(\xi) \\
\vdots & \vdots & \ddots & \vdots \\
f_{N 1}^{j}(\xi) & f_{N 2}^{j}(\xi) & \cdots & f_{N N}^{j}(\xi)
\end{array}\right)
$$

we need to explain that the matrix $M^{*}\left(a^{j} \xi\right) M\left(a^{j}(\xi+2 q \pi)\right)$ is always related to integer $q$, but in order to facilitate mark, q did not appear in the matrix elements $f_{k \ell}^{j}(\xi)$, the same as below.

Assume $\xi \in \mathbb{R}$ satisfies the condition of Lemma 2.10, then there exists $\varepsilon>0$ such that $\xi+2 \pi q \notin \Omega$ and $\varepsilon$ is chosen enough small to ensure $\Omega \neq \mathbb{R}$, where $\Omega=\bigcup_{n \in \mathbb{Z}} a^{n}[\xi-\varepsilon, \xi+\varepsilon]$. According to Lemma 2.9, there exist two wavelet sets $G$ and $H$ with dilation factor a such that $\xi \in G, \xi+2 \pi q \in H$.

Let $\mathrm{E}=\mathrm{G} \cap \Omega, \mathrm{F}=\mathrm{H} \cap \Omega^{\mathrm{c}}$, where $\Omega^{\mathrm{c}}$ is complementary set of $\Omega$. It is obvious that $\Omega$ and $\Omega^{\mathrm{c}}$ are a-dilation invariant, i.e., $a \Omega=\Omega$ and $a \Omega^{\mathfrak{c}}=\Omega^{\mathfrak{c}}$ because of $a \mathbb{R}=\mathbb{R}$.

Let $\widehat{\psi^{1}}=\frac{1}{\sqrt{2 \pi}} \chi_{\mathrm{E}}, \widehat{\psi^{2}}=\frac{1}{\sqrt{2 \pi}} \chi_{\mathrm{F}} . \mathrm{G}$ is a wavelet set with dilation factor $a$, so $|\mathrm{G}|=2 \pi,\{\mathrm{G}+2 \ell \pi: \ell \in \mathbb{Z}\}$ and $\left\{a^{n} G: n \in \mathbb{Z}\right\}$ forms a partition of $\mathbb{R}$. This contains that $E$ has translation invariance of $2 \pi$ and $\left\{a^{n} E: n \in \mathbb{Z}\right\}$ forms a partition of $\Omega$. Therefore, $\psi^{1}$ is a Parseval frame wavelet in $L^{2}(\Omega)$. Similarly, $\psi^{2}$ is a Parseval frame wavelet in $\mathrm{L}^{2}\left(\Omega^{\mathrm{c}}\right)$. So $\left(0, \cdots, 0, \psi^{1}, 0, \cdots, 0, \cdots, \psi^{2}, 0, \cdots, 0\right)$ is a Parseval frame multiwavelet with dilation factor a of length $N$, where $\psi^{1}$ is $\ell$-th component and $\psi^{2}$ is $k$-th component.

$\mathrm{M}(\xi)$ is a matrix Fourier multiwavelet frames multiplier with dilation factor a, let

$$
\left(\widehat{\eta}^{1}(\xi), \widehat{\eta^{2}}(\xi), \cdots, \widehat{\eta^{N_{1}}}(\xi)\right)^{\top}=M(\xi)\left(0, \cdots, 0, \widehat{\psi^{1}}(\xi), 0, \cdots, 0, \cdots, \widehat{\psi^{2}}(\xi), 0, \cdots, 0\right)^{\top} .
$$

Then, $\left(\eta^{1}, \eta^{2}, \ldots, \eta^{N_{1}}\right)$ is also a Parseval frame multiwavelet with dilation factor a, therefore

$$
\begin{aligned}
& 0=\rho_{q}(\xi)=\sum_{\ell=1}^{N_{1}} \sum_{j=0}^{\infty} \widehat{\eta^{\ell}}\left(a^{j} \xi\right) \widehat{\eta^{\ell}\left(a^{j}(\xi+2 \pi q)\right)} \\
& =\sum_{j=0}^{\infty}\left\langle\left(\begin{array}{c}
\vdots \\
\widehat{\psi^{1}}\left(a^{j} \xi\right) \\
\vdots \\
\widehat{\psi^{2}}\left(a^{j} \xi\right) \\
\vdots
\end{array}\right), M^{*}\left(a^{j} \xi\right) M\left(a^{j}(\xi+2 \pi q)\right)\left(\begin{array}{c}
\widehat{\psi^{1}}\left(a^{j}(\xi+2 \pi q)\right) \\
\vdots \\
\widehat{\psi^{2}}\left(a^{j}(\xi+2 \pi q)\right) \\
\vdots
\end{array}\right)\right\rangle .
\end{aligned}
$$

Because $\xi \in \mathrm{E}, \xi+2 \pi \mathrm{q} \in \mathrm{F}, \mathrm{E} \cap \mathrm{F}=\emptyset, \mathrm{E}$ and $\mathrm{F}$ are $2 \pi$-translation disjoint, we can get that $\psi^{1}(\xi)=$ $1, \psi^{1}(\xi+2 \pi q)=0, \psi^{2}(\xi)=0$ and $\psi^{2}(\xi+2 \pi q)=0$. Based on the translation invariance of $\Omega$ and $\Omega^{\mathrm{c}}$, we get that for any $j>0$,

$$
\psi^{1}\left(a^{j} \xi\right)=\psi^{1}\left(a^{j}(\xi+2 \pi q)\right)=\psi^{2}\left(a^{j} \xi\right)=\psi^{2}\left(a^{j}(\xi+2 \pi q)\right)=0 .
$$

Therefore, (2.7) becomes

$$
0=\left\langle\left(\begin{array}{c}
\vdots \\
\widehat{\psi^{1}}\left(a^{j} \xi\right) \\
\vdots \\
\widehat{\psi^{2}}\left(a^{j} \xi\right) \\
\vdots
\end{array}\right),\left(\begin{array}{cccc}
f_{11}^{0}(\xi) & f_{12}^{0}(\xi) & \cdots & f_{1 N}^{0}(\xi) \\
f_{21}^{0}(\xi) & f_{22}^{0}(\xi) & \cdots & f_{2 N}^{0}(\xi) \\
\vdots & \vdots & \ddots & \vdots \\
f_{N 1}^{0}(\xi) & f_{N 2}^{0}(\xi) & \cdots & f_{N N}^{0}(\xi)
\end{array}\right)\left(\begin{array}{c}
\vdots \\
\widehat{\psi^{1}}(\xi+2 \pi q) \\
\vdots \\
\widehat{\psi^{2}}(\xi+2 \pi q) \\
\vdots
\end{array}\right)\right\rangle
$$




$$
=\left\langle\left(\begin{array}{c}
\vdots \\
1 \\
\vdots \\
0 \\
\vdots
\end{array}\right),\left(\begin{array}{cccc}
f_{11}^{0}(\xi) & f_{12}^{0}(\xi) & \cdots & f_{1 N}^{0}(\xi) \\
f_{21}^{0}(\xi) & f_{22}^{0}(\xi) & \cdots & f_{2 N}^{0}(\xi) \\
\vdots & \vdots & \ddots & \vdots \\
f_{N 1}^{0}(\xi) & f_{N 2}^{0}(\xi) & \cdots & f_{N N}^{0}(\xi)
\end{array}\right)\left(\begin{array}{c}
\vdots \\
0 \\
\vdots \\
1 \\
\vdots
\end{array}\right)\right\rangle .
$$

This means that $f_{\ell k}^{0}(\xi)=0, \ell \neq k$. By the arbitrariness of $\ell, k, M^{*}(\xi) M(\xi+2 q \pi)$ is a diagonal matrix. For any $j_{0}>0$, using Lemma 2.9 , there exists $\varepsilon_{j_{0}}>0$ such that $a^{j_{0}} \xi+2 \pi a^{j_{0}} q \notin \Omega_{j_{0}}$, where

$$
\Omega_{j_{0}}=\bigcup_{n \in \mathbb{Z}} a^{n}\left[a^{j_{0}} \xi-\varepsilon_{j_{0}}, a^{j_{0}} \xi+\varepsilon_{j_{0}}\right]
$$

Let $G_{j_{0}}$ and $H_{j_{0}}$ be two wavelet sets, such that $a^{j_{0}} \xi \in G_{j_{0}}, a^{j_{0}} \xi+2 \pi a^{j_{0}} q \in H_{j_{0}}$. Let $E_{j_{0}}=G_{j_{0}} \cap \Omega_{j_{0}}, F_{j_{0}}=$ $\mathrm{H}_{j_{0}} \cap \Omega_{j_{0}}^{c}$, and define $\widehat{\psi_{j_{0}}^{1}}=\frac{1}{\sqrt{2 \pi}} \chi_{\mathrm{E}_{j_{0}}}, \widehat{\psi_{j_{0}}^{2}}=\frac{1}{\sqrt{2 \pi}} \chi_{\mathrm{F}_{0}}$. Based on the above discussions, we obtain that $f_{\ell k}^{j_{0}}(\xi)=0$, when $\ell \neq k$. Utilizing the arbitrariness of $j_{0}$, we have that $j \geqslant 0, q \in \mathbb{Z} \backslash a \mathbb{Z}$, for $\xi \in \mathbb{R}$, thus $M^{*}\left(a^{j} \xi\right) M\left(a^{j}(\xi+2 q \pi)\right)$ is a diagonal matrix.

Remark 2.13. When $a \geqslant 3$, we do not know that whether the conditions (2) and (3) of Theorem 2.1 are the necessary conditions of matrix Fourier multiwavelet frames multiplier. Therefore, when $a \geqslant 3$, the necessary and sufficient conditions of matrix Fourier multiwavelet frames multiplier $M(\xi)$ with dilation factor a are worth further discussion. In fact, sufficient conditions may be more important for constructing a new Parseval multiwavelet frames with dilation factor a.

\section{Numerical example}

Example 3.1. When $a=2$, let $M_{1}(\xi)=\left(\begin{array}{cc}\sin (\xi) & -\cos (\xi) \\ \cos (\xi) & \sin (\xi)\end{array}\right)$, then $M_{1}(\xi)$ is a matrix Fourier frame multiwavelet multiplier with dilation factor 2 . Applying $M_{1}(\xi)$ to Parseval frame multiwavelet $\left(\psi^{1}, \psi^{2}\right)$, where

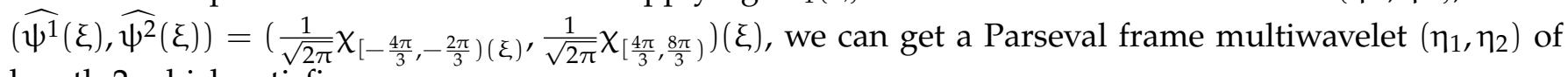
length 2 which satisfies

$$
\left(\begin{array}{c}
\widehat{\eta^{1}}(\xi) \\
\widehat{\eta^{2}}(\xi)
\end{array}\right)=M_{1}(\xi)\left(\begin{array}{c}
\widehat{\psi^{1}}(\xi) \\
\widehat{\psi^{2}}(\xi)
\end{array}\right)=\frac{1}{\sqrt{2 \pi}}\left(\begin{array}{l}
\sin (\xi) \chi_{\left[-\frac{4 \pi}{3},-\frac{2 \pi}{3}\right)}(\xi)-\cos (\xi) \chi_{\left[\frac{4 \pi}{3}, \frac{8 \pi}{3}\right)}(\xi) \\
\cos (\xi) \chi_{\left[-\frac{4 \pi}{3},-\frac{2 \pi}{3}\right)}(\xi)+\sin (\xi) \chi_{\left[\frac{4 \pi}{3}, \frac{8 \pi}{3}\right)}(\xi)
\end{array}\right)
$$

The images of $\left(\widehat{\eta^{1}}, \widehat{\eta^{2}}\right)$ are shown in Figure 1 .
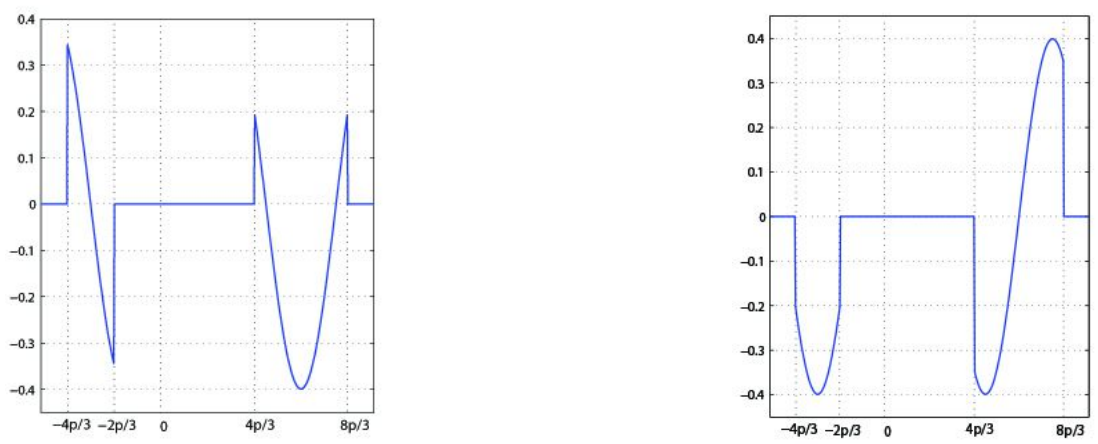

Figure 1: The images of $\widehat{\eta^{1}}, \widehat{\eta^{2}}$. 
Example 3.2. When $a=2$, let $M_{2}(\xi)=\left(\begin{array}{cc}\sin (\xi) & -\cos (\xi) \\ \frac{\sqrt{3}}{2} \cos (\xi) & \frac{\sqrt{3}}{2} \sin (\xi) \\ \frac{1}{2} \cos (\xi) & \frac{1}{2} \sin (\xi)\end{array}\right)$, then $M_{2}(\xi)$ is a matrix Fourier frame multiwavelet multiplier with dilation factor 2. Applying $M_{2}(\xi)$ to Parseval frame multiwavelet $\left(\psi^{1}, \psi^{2}\right)$, where $\left(\widehat{\psi^{1}}(\xi), \widehat{\psi^{2}}(\xi)\right)=\left(\frac{1}{\sqrt{2 \pi}} \chi_{\left[-\frac{4 \pi}{3},-\frac{2 \pi}{3}\right)(\xi)}, \frac{1}{\sqrt{2 \pi}} \chi_{\left[\frac{4 \pi}{3}, \frac{8 \pi}{3}\right)}\right)(\xi)$, we can get a Parseval frame multiwavelet $\left(\eta_{1}, \eta_{2}, \eta_{3}\right)$ of length 2 which satisfies

$$
\left(\begin{array}{c}
\widehat{\eta^{1}}(\xi) \\
\widehat{\eta^{2}}(\xi) \\
\widehat{\eta^{3}}(\xi)
\end{array}\right)=M(\xi)\left(\begin{array}{c}
\widehat{\psi^{1}}(\xi) \\
\widehat{\psi^{2}}(\xi)
\end{array}\right)=\frac{1}{\sqrt{2 \pi}}\left(\begin{array}{c}
\sin (\xi) \chi_{\left[-\frac{4 \pi}{3},-\frac{2 \pi}{3}\right)}(\xi)-\cos (\xi) \chi_{\left[\frac{4 \pi}{3}, \frac{8 \pi}{3}\right)}(\xi) \\
\frac{\sqrt{3}}{2} \cos (\xi) \chi_{\left[-\frac{4 \pi}{3},-\frac{2 \pi}{3}\right)}(\xi)+\frac{\sqrt{3}}{2} \sin (\xi) \chi_{\left[\frac{4 \pi}{3}, \frac{8 \pi}{3}\right)}(\xi) \\
\frac{1}{2} \cos (\xi) \chi_{\left[-\frac{4 \pi}{3},-\frac{2 \pi}{3}\right)}(\xi)+\frac{1}{2} \sin (\xi) \chi_{\left[\frac{4 \pi}{3}, \frac{8 \pi}{3}\right)}(\xi)
\end{array}\right) .
$$

Example 3.3. When $a=2$, let $M_{3}(\xi)=\left(\begin{array}{ccc}\cos (\theta) & \sin (\theta) \cos (\xi) & \sin (\theta) \sin (\xi) \\ \sin (\theta) & -\cos (\theta) \cos (\xi) & -\cos (\theta) \sin (\xi) \\ 0 & \sin (\xi) & -\cos (\xi)\end{array}\right), \xi, \theta \in \mathbb{R}$ are parameters, then $\mathrm{M}_{3}(\xi)$ is a matrix Fourier frame multiwavelet multiplier with dilation factor 2. Applying $M_{3}(\xi)$ to Parseval frame multiwavelet $\left(\psi^{1}, \psi^{2}, \psi^{3}\right)$, where

$$
\left(\widehat{\psi^{1}}(\xi), \widehat{\psi^{2}}(\xi), \widehat{\psi^{3}}(\xi)\right)=\left(\frac{1}{\sqrt{2 \pi}} \chi_{\left[-\frac{32 \pi}{7},-4 \pi\right) \cup\left[-\pi,-\frac{4 \pi}{7}\right)}(\xi), \frac{1}{\sqrt{2 \pi}} \chi_{\left[\frac{4 \pi}{7}, \pi\right)}(\xi), \frac{1}{\sqrt{2 \pi}} \chi_{\left[4 \pi, \frac{32 \pi}{7}\right)}(\xi)\right),
$$

we can get a Parseval frame multiwavelet $\left(\eta_{1}, \eta_{2}, \eta_{3}\right)$ of length 3 which satisfies

$$
\begin{aligned}
\left(\begin{array}{c}
\widehat{\eta^{1}}(\xi) \\
\widehat{\eta^{2}}(\xi) \\
\widehat{\eta^{3}}(\xi)
\end{array}\right) & =M_{3}(\xi)\left(\begin{array}{c}
\widehat{\psi^{1}}(\xi) \\
\widehat{\psi^{2}}(\xi) \\
\widehat{\psi^{3}}(\xi)
\end{array}\right) \\
& =\frac{1}{\sqrt{2 \pi}}\left(\begin{array}{c}
\cos (\theta) x_{\left[-\frac{32 \pi}{7},-4 \pi\right) \cup\left[-\pi,-\frac{4 \pi}{7}\right)}(\xi)+\sin (\theta) \cos (\xi) x_{\left[\frac{4 \pi}{7}, \pi\right)}(\xi)+\sin (\theta) \sin (\xi) \chi_{\left[4 \pi, \frac{32 \pi}{7}\right)}(\xi) \\
\sin (\theta) \chi_{\left[-\frac{32 \pi}{7},-4 \pi\right) \cup\left[-\pi,-\frac{4 \pi}{7}\right)}(\xi)-\cos (\theta) \cos (\xi) x_{\left[\frac{4 \pi}{7}, \pi\right)}(\xi)-\cos (\theta) \sin (\xi) \chi_{\left[4 \pi, \frac{32 \pi}{7}\right)}(\xi) \\
\sin (\xi) \chi_{\left[\frac{4 \pi}{7}, \pi\right)}(\xi)-\cos (\xi) \chi_{\left[4 \pi, \frac{32 \pi}{7}\right)}(\xi)
\end{array}\right) .
\end{aligned}
$$

The images of $\left(\widehat{\eta^{1}}, \widehat{\eta^{2}}, \widehat{\eta^{3}}\right)$ are shown in Figure 2. It is important to state that $M_{3}(\xi)$ is also a matrix Fourier multiwavelet frame multiplier with dilation factor 3.
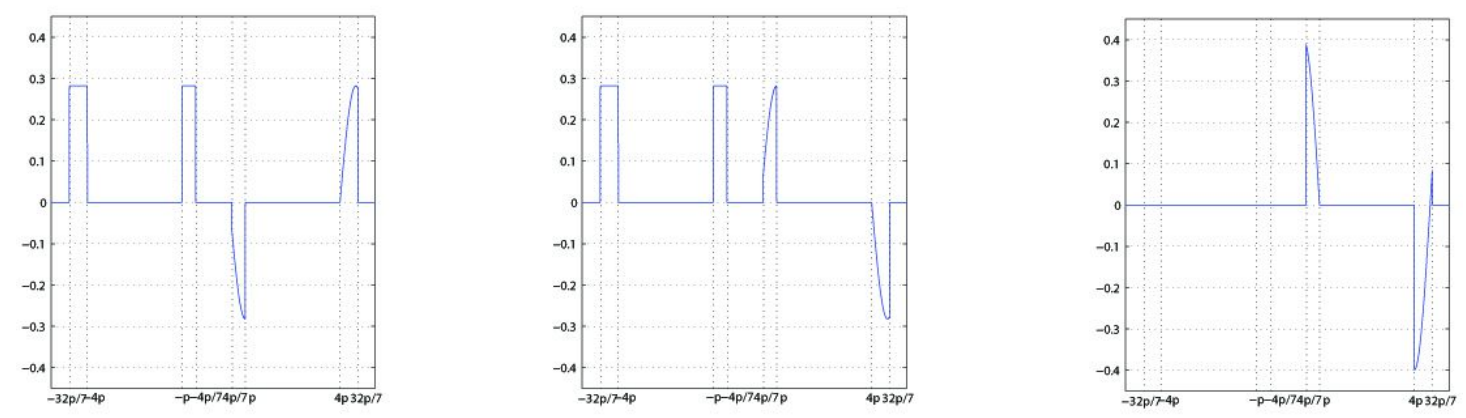

Figure 2: The images of $\widehat{\eta^{1}}, \widehat{\eta^{2}}, \widehat{\eta^{3}}$.

Example 3.4. When $a=3$, let $M_{4}(\xi)=\left(\begin{array}{ccc}e^{i \xi} & 0 & 0 \\ 0 & \cos (\xi) & \sin (\xi) \\ 0 & -\sin (\xi) & \cos (\xi)\end{array}\right), \xi \in \mathbb{R}$, then $M_{4}(\xi)$ is a matrix Fourier frame multiwavelet multiplier with dilation factor 3. Apply $M_{4}(\xi)$ to Parseval frame multiwavelet 
$\left(\psi^{1}, \psi^{2}, \psi^{3}\right)$, where $\left(\widehat{\psi^{1}}(\xi), \widehat{\psi^{2}}(\xi), \widehat{\psi^{3}}(\xi)\right)=\left(\frac{1}{\sqrt{2 \pi}} \chi_{\left[-\frac{3 \pi}{2},-\frac{\pi}{2}\right)}(\xi), \frac{1}{\sqrt{2 \pi}} \chi_{\left[\frac{\pi}{4}, \frac{\pi}{2}\right)}(\xi), \frac{1}{\sqrt{2 \pi}} \chi_{\left[\frac{3 \pi}{2}, \frac{9 \pi}{4}\right)}(\xi)\right)$. We can get the length of 3 and 3 scale Parseval frame multiwavelet $\left(\eta_{1}, \eta_{2}, \eta_{3}\right)$ satisfies

$$
\left(\begin{array}{c}
\widehat{\eta^{1}}(\xi) \\
\widehat{\eta^{2}}(\xi) \\
\widehat{\eta^{3}}(\xi)
\end{array}\right)=M(\xi)\left(\begin{array}{c}
\widehat{\psi^{1}}(\xi) \\
\widehat{\psi^{2}}(\xi) \\
\widehat{\psi^{3}}(\xi)
\end{array}\right)=\frac{1}{\sqrt{2 \pi}}\left(\begin{array}{c}
e^{\frac{i \xi}{2}} \chi_{\left[-\frac{3 \pi}{2},-\frac{1 \pi}{2}\right)}(\xi) \\
\cos (\xi) \chi_{\left[\frac{\pi}{4}, \frac{\pi}{2}\right)}(\xi)+\sin (\xi) \chi_{\left[\frac{3 \pi}{2}, \frac{9 \pi}{4}\right)}(\xi) \\
-\sin (\xi) \chi_{\left[\frac{\pi}{4}, \frac{\pi}{2}\right)}(\xi)+\cos (\xi) \chi_{\left[\frac{3 \pi}{2}, \frac{9 \pi}{4}\right)}(\xi)
\end{array}\right) .
$$

The images of $\left(\widehat{\eta^{1}}, \widehat{\eta^{2}}, \widehat{\eta^{3}}\right)$ are shown in Figures 3 and 4 .
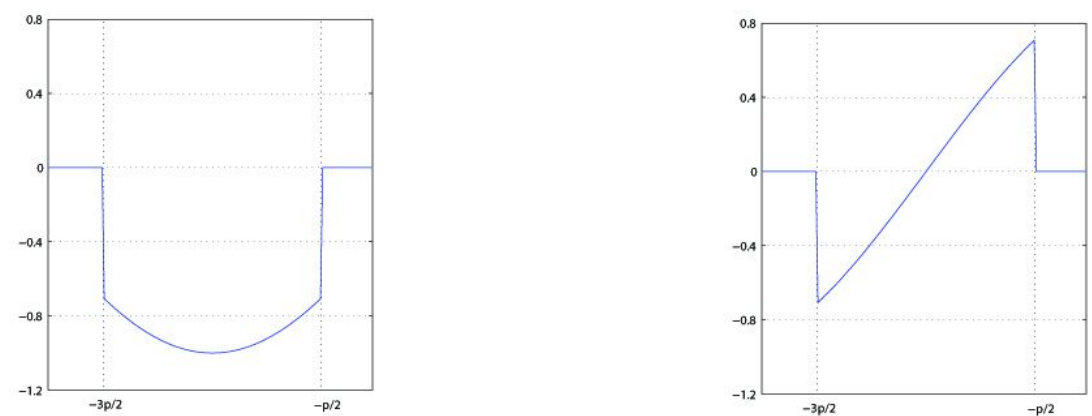

Figure 3: The images of the imaginary part and real part of $\widehat{\eta^{1}}$.
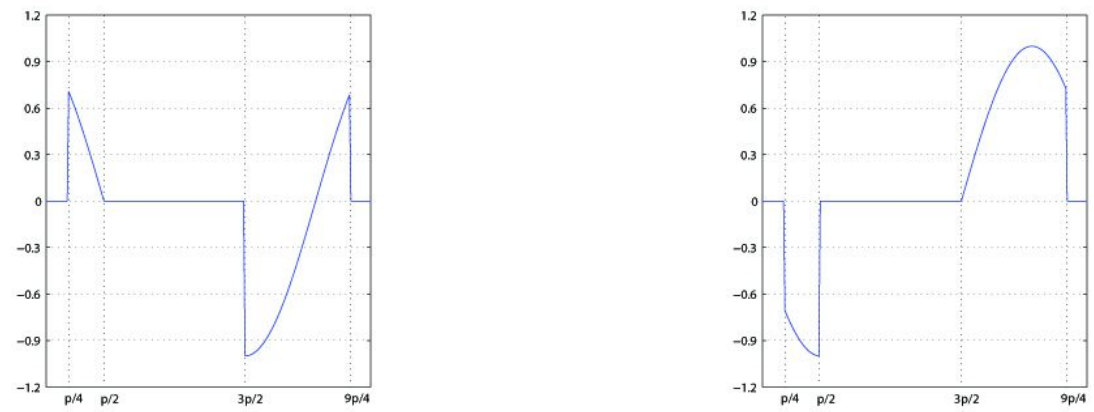

Figure 4: The image of $\widehat{\eta^{2}}, \widehat{\eta^{3}}$.

Example 3.5. When $a=3$, let $M_{5}(\xi)=\left(\begin{array}{ccc}e^{i \xi} & 0 & 0 \\ 0 & \frac{\sqrt{2}}{2} e^{-i \xi} & 0 \\ 0 & \frac{\sqrt{2}}{2} \cos (\xi) & \sin (\xi) \\ 0 & -\frac{\sqrt{2}}{2} \sin (\xi) & \cos (\xi)\end{array}\right), \xi \in \mathbb{R}$, then $M_{5}(\xi)$ is a matrix Fourier frame multiwavelet multiplier with dilation factor 3. Applying $M_{5}(\xi)$ to Parseval frame multiwavelet $\left(\psi^{1}, \psi^{2}, \psi^{3}\right)$ with dilation factor 3 , where

$$
\left(\widehat{\psi^{1}}(\xi), \widehat{\psi^{2}}(\xi), \widehat{\psi^{3}}(\xi)\right)=\left(\frac{1}{\sqrt{2 \pi}} \chi_{\left[-\frac{3 \pi}{2},-\frac{\pi}{2}\right)}(\xi), \frac{1}{\sqrt{2 \pi}} \chi_{\left[\frac{\pi}{4}, \frac{\pi}{2}\right)}(\xi), \frac{1}{\sqrt{2 \pi}} \chi_{\left[\frac{3 \pi}{2}, \frac{9 \pi}{4}\right)}(\xi)\right),
$$

we can get a Parseval frame multiwavelet $\left(\eta_{1}, \eta_{2}, \eta_{3}, \eta_{4}\right)$ which satisfies

$$
\left(\begin{array}{c}
\widehat{\eta^{1}}(\xi) \\
\widehat{\eta^{2}}(\xi) \\
\widehat{\eta^{3}}(\xi) \\
\widehat{\eta^{4}}(\xi)
\end{array}\right)=M(\xi)\left(\begin{array}{c}
\widehat{\psi^{1}}(\xi) \\
\widehat{\psi^{2}}(\xi) \\
\widehat{\psi^{3}}(\xi)
\end{array}\right)=\frac{1}{\sqrt{2 \pi}}\left(\begin{array}{c}
e^{i \xi} \chi_{\left[-\frac{3 \pi}{2},-\frac{1 \pi}{2}\right)}(\xi) \\
\frac{\sqrt{2}}{2} e^{-i \xi} \chi_{\left[\frac{\pi}{4}, \frac{\pi}{2}\right)}(\xi) \\
\frac{\sqrt{2}}{2} \cos (\xi) \chi_{\left[\frac{\pi}{4}, \frac{\pi}{2}\right)}(\xi)+\sin (\xi) \chi_{\left[\frac{3 \pi}{2}, \frac{9 \pi}{4}\right)}(\xi) \\
-\frac{\sqrt{2}}{2} \sin (\xi) \chi_{\left[\frac{\pi}{4}, \frac{\pi}{2}\right)}(\xi)+\cos (\xi) \chi_{\left[\frac{3 \pi}{2}, \frac{9 \pi}{4}\right)}(\xi)
\end{array}\right)
$$


Example 3.6. When $a=3$, let $M_{6}(\xi)=\left(\begin{array}{ccc}\sin (\xi) & \cos (\xi) \sin (\theta) & \cos (\xi) \cos (\theta) \\ -\cos (\xi) & \sin (\xi) \sin (\theta) & \sin (\xi) \cos (\theta) \\ 0 & \cos (\theta) & -\sin (\theta)\end{array}\right), \theta \in \mathbb{R}$, then $M_{6}(\xi)$ is a matrix Fourier frame multiwavelet multiplier with dilation 3. Applying $M_{6}(\xi)$ to Parseval frame multiwavelet $\left(\psi^{1}, \psi^{2}, \psi^{3}\right)$ with dilation factor 3 , where

$$
\left(\widehat{\psi^{1}}(\xi), \widehat{\psi^{2}}(\xi), \widehat{\psi^{3}}(\xi)\right)=\left(\frac{1}{\sqrt{2 \pi}} \chi_{\left[-\frac{3 \pi}{2},-\frac{\pi}{2}\right)}(\xi), \frac{1}{\sqrt{2 \pi}} \chi_{\left[\frac{\pi}{4}, \frac{\pi}{2}\right)}(\xi), \frac{1}{\sqrt{2 \pi}} \chi_{\left[\frac{3 \pi}{2}, \frac{9 \pi}{4}\right)}(\xi)\right),
$$

we can get a Parseval frame multiwavelet $\left(\eta_{1}, \eta_{2}, \eta_{3}\right)$ which satisfies

$$
\left(\begin{array}{c}
\widehat{\eta^{1}}(\xi) \\
\widehat{\eta^{2}}(\xi) \\
\widehat{\eta^{3}(\xi)}
\end{array}\right)=\frac{1}{\sqrt{2 \pi}}\left(\begin{array}{c}
\left.\sin (\xi) \chi_{\left[-\frac{3 \pi}{2},-\frac{\pi}{2}\right)}(\xi)+\cos (\xi) \sin (\theta) \chi_{\left[\frac{\pi}{4}, \frac{\pi}{2}\right)}(\xi)+\cos (\xi) \cos (\theta) \chi_{\left[\frac{3 \pi}{2},\right.}, \frac{9 \pi}{4}\right) \\
-\cos (\xi) \chi_{\left[-\frac{3 \pi}{2},-\frac{\pi}{2}\right)}(\xi)+\sin (\xi) \sin (\theta) \chi_{\left[\frac{\pi}{4}, \frac{\pi}{2}\right)}(\xi)+\sin (\xi) \cos (\theta) \chi_{\left[\frac{3 \pi}{2}, \frac{9 \pi}{4}\right)}(\xi) \\
\cos (\theta) \chi_{\left[\frac{\pi}{4}, \frac{\pi}{2}\right)}(\xi)-\sin (\theta) \chi_{\left[\frac{3 \pi}{2}, \frac{9 \pi}{4}\right)}(\xi)
\end{array}\right) .
$$

The images of $\left(\widehat{\eta^{1}}, \widehat{\eta^{2}}, \widehat{\eta^{3}}\right)$ are shown in Figure 5 .
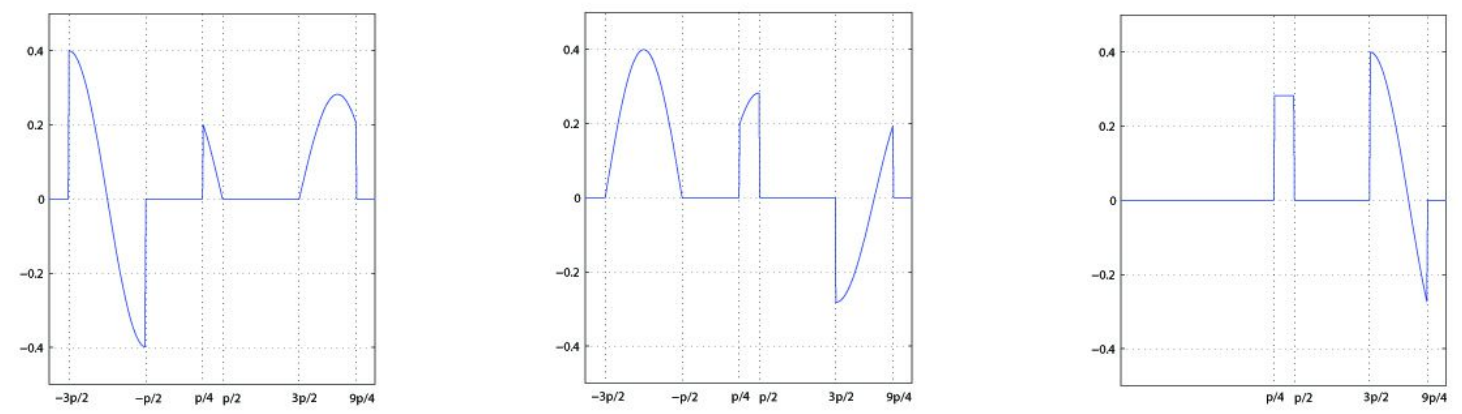

Figure 5: The images of $\widehat{\eta_{1}}, \widehat{\eta_{2}}, \widehat{\eta_{3}}$.

Example 3.7. When $a=4$, let

$$
M_{7}(\xi)=\left(\begin{array}{cccc}
\cos (\xi) \sin (\theta) & \cos (\xi) \cos (\theta) & \sin (\xi) \cos (\theta) & \sin (\xi) \sin (\theta) \\
\sin (\xi) \cos (\theta) & -\sin (\xi) \sin (\theta) & -\cos (\xi) \sin (\theta) & \cos (\xi) \cos (\theta) \\
\sin (\xi) \sin (\theta) & \sin (\xi) \cos (\theta) & -\cos (\xi) \cos (\theta) & -\cos (\xi) \sin (\theta) \\
\cos (\xi) \cos (\theta) & -\cos (\xi) \sin (\theta) & \sin (\xi) \sin (\theta) & -\sin (\xi) \cos (\theta)
\end{array}\right)
$$

$\xi, \theta \in \mathbb{R}$, then $M_{7}(\xi)$ is a matrix Fourier frame multiwavelet multiplier with dilation factor 4. Applying $M_{7}(\xi)$ to Parseval frame multiwavelet $\left(\psi^{1}, \psi^{2}, \psi^{3}, \psi^{4}\right)$, where

$$
\left(\widehat{\psi^{1}}(\xi), \widehat{\psi^{2}}(\xi), \widehat{\psi^{3}}(\xi), \widehat{\psi^{4}}(\xi)\right)=\frac{1}{\sqrt{2 \pi}}\left(\chi_{\left[-\frac{8 \pi}{5},-\frac{2 \pi}{5}\right)}(\xi), \chi_{\left[\frac{2 \pi}{15}, \frac{2 \pi}{5}\right)}(\xi), \chi_{\left[\frac{8 \pi}{5}, 2 \pi\right)}(\xi), \chi_{\left[2 \pi, \frac{32 \pi}{15}\right)}(\xi)\right),
$$

we can get a Parseval frame multiwavelet $\left(\eta_{1}, \eta_{2}, \eta_{3}, \eta_{4}\right)$ which satisfies

$$
\begin{aligned}
& \sqrt{2 \pi}\left(\begin{array}{c}
\widehat{\eta^{1}}(\xi) \\
\widehat{\eta^{2}}(\xi) \\
\widehat{\eta^{3}}(\xi) \\
\widehat{\eta^{4}}(\xi)
\end{array}\right)= \\
& \left(\begin{array}{l}
\cos (\xi) \sin (\theta) \chi_{\left[-\frac{8 \pi}{5},-\frac{2 \pi}{5}\right)}(\xi)+\cos (\xi) \cos (\theta) \chi_{\left[\frac{2 \pi}{15}, \frac{2 \pi}{5}\right)}(\xi)+\sin (\xi) \cos (\theta) \chi_{\left[\frac{8 \pi}{5}, 2 \pi\right)}(\xi)+\sin (\xi) \sin (\theta) \chi_{\left[2 \pi, \frac{32 \pi}{15}\right)}(\xi) \\
\sin (\xi) \cos (\theta) \chi_{\left[-\frac{8 \pi}{5},-\frac{2 \pi}{5}\right)}(\xi)-\sin (\xi) \sin (\theta) \chi_{\left[\frac{2 \pi}{15}, \frac{2 \pi}{5}\right)}(\xi)-\cos (\xi) \sin (\theta) \chi_{\left[\frac{8 \pi}{5}, 2 \pi\right)}(\xi)+\cos (\xi) \cos (\theta) \chi_{\left[2 \pi, \frac{2 \pi}{15}\right)}(\xi) \\
\sin (\xi) \sin (\theta) \chi_{\left[-\frac{8 \pi}{5},-\frac{2 \pi}{5}\right)}(\xi)+\sin (\xi) \cos (\theta) \chi_{\left[\frac{2 \pi}{15}, \frac{2 \pi}{5}\right)}(\xi)-\cos (\xi) \cos (\theta) \chi_{\left[\frac{8 \pi}{5}, 2 \pi\right)}(\xi)-\cos (\xi) \sin (\theta) \chi_{\left[2 \pi, \frac{32 \pi}{15}\right)}(\xi) \\
\cos (\xi) \cos (\theta) \chi_{\left[-\frac{8 \pi}{5},-\frac{2 \pi}{5}\right)}(\xi)-\cos (\xi) \sin (\theta) \chi_{\left[\frac{2 \pi}{15}, \frac{2 \pi}{5}\right)}(\xi)+\sin (\xi) \sin (\theta) \chi_{\left[\frac{8 \pi}{5}, 2 \pi\right)}(\xi)-\sin (\xi) \cos (\theta) \chi_{\left[2 \pi, \frac{32 \pi}{15}\right)}(\xi)
\end{array}\right) .
\end{aligned}
$$


The images of $\left(\widehat{\eta^{1}}, \widehat{\eta^{2}}, \widehat{\eta^{3}}, \widehat{\eta^{4}}\right)$ are shown in Figure 6 .
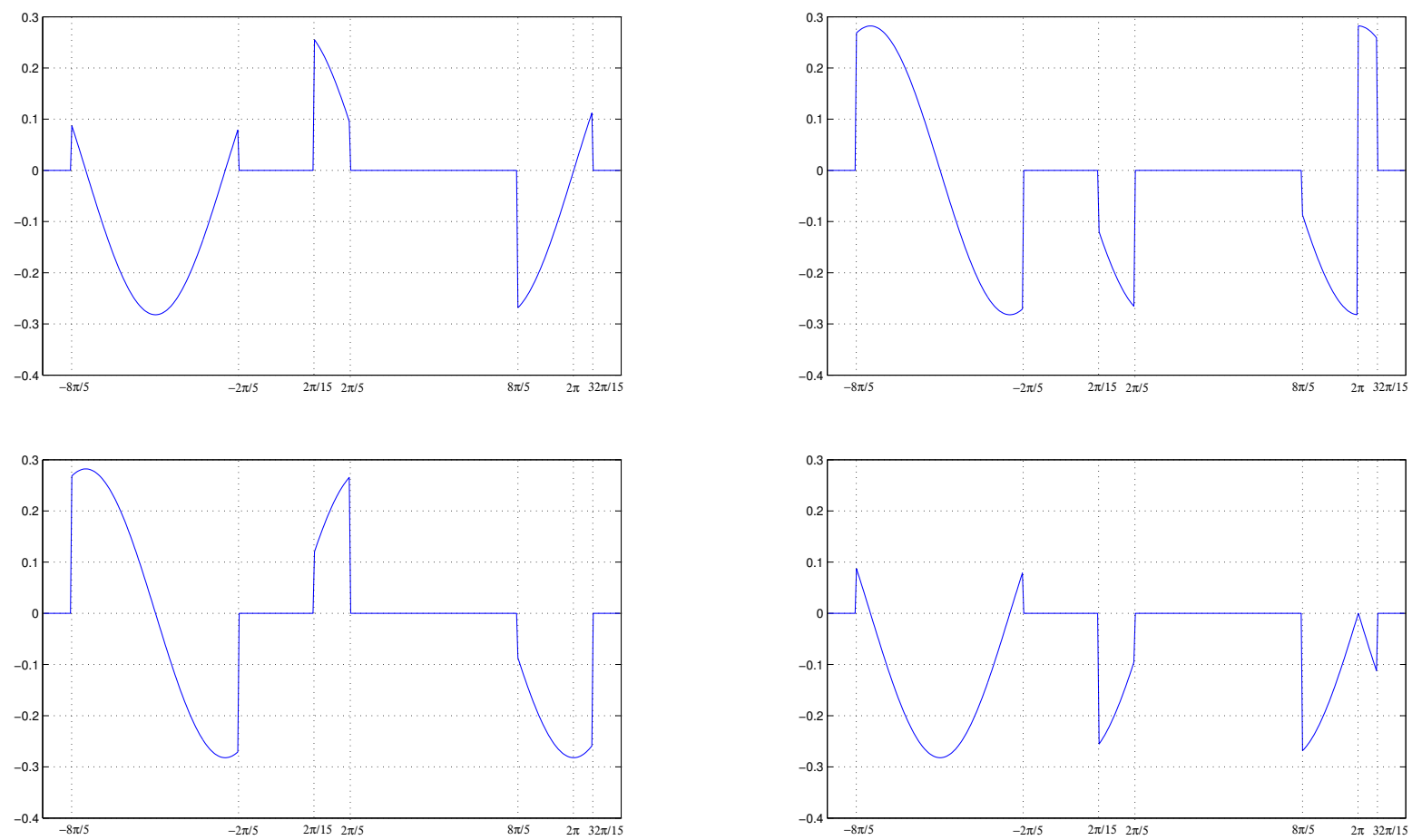

Figure 6: The images of $\widehat{\eta^{1}}, \widehat{\eta^{2}}, \widehat{\eta^{3}}, \widehat{\eta^{4}}$.

\section{Acknowledgment}

This work is supported by National Natural Science Foundation of China (Grant no. 11761001) and Science Foundation of North Minzu University (Grant nos. 2016SXKY04 and 2017KJ36).

\section{References}

[1] D. Bakić, I. Krishtal, E. N. Wilson, Parseval frame wavelets with $\mathrm{E}_{n}^{(2)}$-dilations, Appl. Comput. Harmon. Anal., 19 (2005), 386-431. 1

[2] M. Bownik, A characterization of affine dual frames in $\mathrm{L}^{2}\left(\mathbb{R}^{n}\right)$, Appl. Comput. Harmon. Anal., 8 (2000), 203-221. 1.1, 1.2

[3] M. Bownik, Connectivity and density in the set of framelets, Math. Res. Lett., 14 (2007), 285-293. 2

[4] M. Bownik, The closure of the set of tight frame wavelets, Acta Appl. Math., 107 (2009), 195-201. 2

[5] J.-F. Cheng, D.-F. Li, The characterization of MRA E-tight frame wavelet, Acta Math. Sin. Chin. Ser., 51 (2008), 877-888. 1

[6] X.-D. Dai, D. R. Larson, Wandering vectors for unitary systems and orthogonal wavelets, Mem. Amer. Math. Soc., 134 (1998), 68 pages. 1

[7] X.-D. Dai, D. R. Larson, D. M. Speegle, Wavelet sets in $\mathbb{R}^{n}$, J. Fourier Anal. Appl., 3 (1997), 451-456. 2, 2, 2.9

[8] D.-G. Han, D. R. Larson, Wandering vector multipliers for unitary groups, Trans. Amer. Math. Soc., 352 (2001), $3347-$ 3370. 1

[9] D.-G. Han, D. R. Larson, On the orthogonality of frames and the density and connectivity of wavelet frames, Acta Appl. Math., 107 (2009), 211-222. 2

[10] D.-G. Han, D. R. Larson, Unitary systems and Bessel generator multipliers, Wavelets and multiscale analysis, Appl. Numer. Harmon. Anal., Birkhäuser/Springer, New York, (2011), 131-150. 1

[11] Y.-D. Huang, N. Sun, Characterizations of A-Parseval frame wavelet, Acta Math. Sin. Chin. Ser., 54 (2011), 767-790.

[12] Y.-D. Huang, F.-J. Zhu, Characterization of matrix Fourier multipliers for A-dilation Parseval multi-wavelet frames, Int. J. Wavelets Multiresolut. Inf. Process., 13 (2015), 22 pages. 1 
[13] Y.-Z. Li, On a class of bidimensional nonseparable wavelet multipliers, J. Math. Anal. Appl., 270 (2002), 543-560. 1

[14] Z.-Y. Li, D.-G. Han, Matrix Fourier multipliers for Parseval multi-wavelet frames, Appl. Comput. Harmon. Anal., 35 (2013), 407-418. 1, 1, 1.4, 1.6, 2.2, 2

[15] Z.-Y. Li, X.-L. Shi, Parseval frame wavelet multipliers in $\mathrm{L}^{2}\left(\mathbb{R}^{\mathrm{d}}\right)$, Chin. Ann. Math. Ser. B, 33 (2012), 949-960. 1

[16] R. Liang, Some properties of wavelets, Thesis (Ph.D.)-The University of North Carolina at Charlotte, ProQuest LLC, Ann Arbor, MI, (1998). 1, 1.3

[17] M. Paluszyński, H. Šikić, G. Weiss, S.-L. Xiao, Generalized low pass filters and MRA frame wavelets, J. Geom. Anal., 11(2011), 311-342. 1

[18] The Wutam Consortium, Basic properties of wavelets, J. Fourier Anal. Appl., 4 (1998), 575-594. 1, 1.3 